\title{
FLT3-ITDos and FLT3-ITDon'ts: navigating maintenance therapy in FLT3-ITD-positive acute myeloid leukemia following stem cell transplantation
}

\author{
Brittany Knick Ragon ${ }^{1}{ }^{1}$
}

Received: 23 November 2020 / Revised: 11 February 2021 / Accepted: 3 March 2021 / Published online: 28 April 2021

(c) The Author(s), under exclusive licence to Springer Nature Limited 2021

Emergence of understanding the critical implication of an internal tandem duplication (ITD) in FMS-like tyrosine kinase-3 (FLT3) in acute myeloid leukemia (AML) has transformed the approach to the high-risk group of patients carrying this activating mutation [1]. With FLT3-ITD recognized in $13-30 \%$ of AML patients, it is the most common mutation detected in this population [2]. When FLT3-ITD is identified in AML patients, chances for favorable outcomes are greatly diminished with increased rates of relapse and decreased overall survival (OS) [3]. Fortunately, as we have now entered the era of targeted therapeutics, progress has been made in the management and subsequent outcomes for patients with FLT3-ITDpositive AML.

OS in AML patients with FLT3-ITD has improved with the introduction of FLT3 inhibitors to intensive induction and consolidation regimens. Midostaurin represents one multitargeted kinase inhibitor now approved for inclusion with intensive induction for FLT3-ITD AML based on the phase 3 results from the RATIFY trial [4]. Importantly, the RATIFY trial investigated midostaurin combined with intensive chemotherapy followed by single-agent maintenance therapy and did not allow continued midostaurin for patients who proceeded to allogeneic hematopoietic stem cell transplant (HSCT).

Although the role of HSCT as consolidation in FLT3ITD-positive AML was initially debated secondary to varying results from several studies, more recent reports have confirmed improvement in relapse free survival (RFS) and OS following HSCT compared to those who did not

Brittany Knick Ragon

brittany.ragon@atriumhealth.org

1 Department of Hematologic Oncology and Blood Disorders, Levine Cancer Institute, Atrium Health, Charlotte, NC, USA receive a transplant $[5,6]$. With clear demonstration of the utility of FLT3 inhibition prior to allogeneic HSCT along with reasonable tolerability of these agents, the value of FLT3 inhibitor maintenance following HSCT is now being explored. Unlike the RATIFY trial, the AMLSG 16-10 phase 2 trial of intensive chemotherapy combined with midostaurin followed by allogeneic HSCT included singleagent midostaurin maintenance for 12 months after transplantation [7]. Event free survival was increased compared to historical controls in the midostaurin treated patients, but the contribution of maintenance midostaurin post-HSCT was not entirely explicated since survival improvement may have been related to the requirement of complete remission prior to receiving HSCT [7]. The randomized phase 2 SORMAIN trial compared FLT3-ITD AML patients assigned to sorafenib compared to placebo for up to 24 months post-HSCT. Despite the study being terminated prematurely due to low accrual, survival favored sorafenib maintenance with a median follow-up duration of 55.1 months [8]. Xuan et al. performed a phase 3 trial randomizing sorafenib maintenance to non-maintenance post-HSCT and determined a decreased 1-year cumulative incidence of relapse in the sorafenib group [9]. The acute leukemia working party of the European Society for Blood and Marrow Transplant (EBMT) explored a retrospective registry-based multicenter analysis demonstrating improved OS in patients receiving post-HSCT sorafenib maintenance [10]. Based on these and several other studies, the EBMT has released a clinical practice recommendation advising post-transplant systemic FLT3 inhibitor maintenance therapy, with sorafenib as the preferred option [11].

In this issue, Maziarz et al. detail results from the RADIUS trial, the first randomized study of maintenance therapy with midostaurin following allogeneic HSCT. Designed as a phase 2, open-label trial evaluating standard of care (SOC) with or without midostaurin in FLT3-ITDpositive AML patients following allogeneic HSCT, RFS at 
18 months served as the primary endpoint. Notably, SOC excluded alternative tyrosine kinase inhibitor (TKI) therapy, with anti-infective and graft versus host disease therapy directed by institutional guidelines. Patients were required to be in first complete remission prior to HSCT, received protocol-specified conditioning, and were then enrolled after engraftment with 1:1 randomization within 28-60 days following transplantation. The study was not sufficiently powered to detect a statistical difference between treatment arms with a slight improvement in RFS at 18 months, 89\% in the midostaurin treated arm versus $76 \%$ with SOC (HR, 0.46 [95\% CI, 0.12-1.86]; $P=0.27$ ). However, the trial did achieve the expected $54 \%$ relative reduction in the risk of relapse 18 months post-transplant and an overall trend toward benefit in the midostaurin arm. Several limitations in interpreting these findings include the likelihood that most patients did not receive a TKI prior to transplantation, which is now SOC for this population. Additionally, there is no measure of molecular minimal residual disease prior to or following HSCT, which might be a critical factor contributing to RFS and also may help to clarify appropriate patients for post-transplant maintenance therapy. Additional investigations are needed to support and provide current context for these results.

An effort by the investigators to distinguish patients that might benefit most from maintenance FLT3 inhibition did serve as a unique study highlight; the authors attempted to correlate a measure of FLT3 functional status to RFS. FLT3 phosphorylation levels were used to demonstrate extent of inhibition of FLT3 activity. Inhibition of FLT3 phosphorylation to less than $70 \%$ of baseline (representing effective inhibition of FLT3 activity) was associated with improved RFS and OS. Nearly half of the midostaurin treated patients achieved this degree of inhibition, and this measure might be useful in practice to better guide maintenance therapy.

To build upon this work, the Blood and Marrow Transplant Clinical Trials Network protocol 1506 serves as the first phase 3 randomized and double-blind trial of maintenance therapy with selective FLT3 inhibitor gilteritinib versus placebo in FLT3-ITD AML patients in first complete remission following HSCT [12]. This trial will include minimal residual disease status and is expected to further elucidate the role of FLT3 inhibitor maintenance posttransplant.

So, when we have a FLT3-ITD-positive AML patient, what do we do, and what don't we do? When do we give maintenance, and when do we not? As we have seen the OS of this high-risk group of patients extend over the past several years, and despite lack of clarity as to the extent various interventions have contributed to this finding, there is growing evidence that the use of a FLT3 inhibitor in certain scenarios following HSCT is the correct path forward. While we have not yet identified the precise groups of patients for which FLT3 inhibitor maintenance is most beneficial, current and future studies will clarify this. There is certainly more to learn regarding the role of maintenance FLT3 inhibition post-HSCT. For now, what we should do?

(1) Do use FLT3 inhibitors upfront in our FLT3-ITD AML patients.

(2) Do strongly consider consolidation with allogeneic HSCT for these patients if considered fit enough to proceed.

(3) Do pursue wisely designed maintenance trials powered to determine which patients will benefit most from this strategy.

And what are the don'ts?

(1) Do not assume your FLT3-mutated AML patients will have a bad outcome; there is continued improvement in survival among this population secondary to better understanding and utilization of targeted therapies and transplantation.

(2) Do not consider all FLT3-mutated AML patients equivalent in their risk of relapse; we are learning more regarding the presence or absence of molecular minimal residual disease following treatment initiation and the impact of mutational burden in outcomes with therapy. Eventually, having a tailored approach to individual patients is anticipated, and it can only be assumed that outcomes for these patients will continue to improve.

(3) Do not give up on the idea of maintenance FLT3 inhibition; in time, we will know just what to FLT3ITDo.

\section{Compliance with ethical standards}

Conflict of interest The author declares no competing interests.

Publisher's note Springer Nature remains neutral with regard to jurisdictional claims in published maps and institutional affiliations.

\section{References}

1. Nakao M, Yokota S, Iwai T, Kaneko H, Horiike S, Kashima K, et al. Internal tandem duplication of the flt 3 gene found in acute myeloid leukemia. Leukemia. 1996;10:1911-8.

2. D. Kottaridis P, Gale RE, Linch DC. Flt3 mutations and leukaemia. Br J Haematol. 2003;122:523-38. https://doi.org/10.1046/j.13652141.2003.04500.x.

3. Daver N, Schlenk RF, Russell NH, Levis MJ. Targeting FLT3 mutations in AML: review of current knowledge and evidence. Leukemia. 2019;33:299-312. https://doi.org/10.1038/s41375018-0357-9. 
4. Stone RM, Mandrekar SJ, Sanford BL, Laumann K, Geyer S, Bloomfield $\mathrm{CD}$, et al. Midostaurin plus chemotherapy for acute myeloid leukemia with a FLT3 mutation. N. Engl J Med. 2017;377:454-64. https://doi.org/10.1056/NEJMoa1614359.

5. DeZern AE, Sung A, Kim S, Smith BD, Karp JE, Gore SD, et al. Role of allogeneic transplantation for FLT3/ITD acute myeloid leukemia: outcomes from 133 consecutive newly diagnosed patients from a single institution. Biol Blood Marrow Transpl. 2011;17:1404-9. https://doi.org/10.1016/j.bbmt.2011.02.003.

6. Oran B, Cortes J, Beitinjaneh A, Chen HC, de Lima M, Patel K, et al. Allogeneic transplantation in first remission improves outcomes irrespective of FLT3-ITD allelic ratio in FLT3-ITDpositive acute myelogenous leukemia. Biol Blood Marrow Transpl. 2016;22:1218-26. https://doi.org/10.1016/j.bbmt.2016. 03.027.

7. Schlenk RF, Weber D, Fiedler W, Salih HR, Wulf G, Salwender $\mathrm{H}$, et al. Midostaurin added to chemotherapy and continued single-agent maintenance therapy in acute myeloid leukemia with FLT3-ITD. Blood. 2019;133:840-51. https://doi.org/10.1182/ blood-2018-08-869453.

8. Burchert A, Bug G, Fritz LV, Finke J, Stelljes M, Röllig C, et al. Sorafenib maintenance after allogeneic hematopoietic stem cell transplantation for acute myeloid leukemia With FLT3-internal tandem duplication mutation (SORMAIN). J Clin Oncol. 2020;38: 2993-3002. https://doi.org/10.1200/jco.19.03345.
9. Xuan L, Wang Y, Huang F, Fan Z, Xu Y, Sun J, et al. Sorafenib maintenance in patients with FLT3-ITD acute myeloid leukaemia undergoing allogeneic haematopoietic stem-cell transplantation: an open-label, multicentre, randomised phase 3 trial. Lancet Oncol. 2020;21:1201-12. https://doi.org/10.1016/S1470-2045(20)30455-1.

10. Ali B, Myriam L, Giorgia B, Azedine D, Edouard F, William A, et al. Allogeneic stem cell transplantation for FLT3-mutated acute myeloid leukemia: in vivo T-cell depletion and posttransplant sorafenib maintenance improve survival. A Retrospective Acute Leukemia Working Party-European Society for Blood and Marrow Transplant Study. Clin Hematol Int. 2019;1:58-74. https://doi.org/ 10.2991/chi.d.190310.001.

11. Bazarbachi A, Bug G, Baron F, Brissot E, Ciceri F, Dalle IA, et al. Clinical practice recommendation on hematopoietic stem cell transplantation for acute myeloid leukemia patients with FLT3internal tandem duplication: a position statement from the Acute Leukemia Working Party of the European Society for Blood and Marrow Transplantation. Haematologica. 2020;105:1507-16. https://doi.org/10.3324/haematol.2019.243410.

12. Levis MJ, Hamadani M, Logan BR, Rosales M, Delgado D, Bahceci E, et al. BMT CTN Protocol 1506: A Phase 3 Trial of Gilteritinib As Maintenance Therapy after Allogeneic Hematopoietic Stem Cell Transplantation in Patients with FLT3-ITD+ AML. Blood. 2019;134:4602. https://doi.org/10.1182/blood2019-124322. 